\title{
Qualitative Regressions: Model the uncertainty in your (qualitative) data to make better inferences
}

\author{
Didier Ruedin (University of Neuchâtel and University of the Witwatersrand)
}

28 March 2017 - Early Draft

Objective: Make better inferences by modelling the uncertainty in your (qualitative) data. Methods: Use regression analysis with qualitative data. Use standard statistical distributions to express uncertainty in the data, then sample from these distributions to create multiple data sets, which is then the same 'problem' as multiple imputations for missing data. Results: I show that this approach is feasible. Conclusion: It is possible to run regression analyses on qualitative data, and we can use information on uncertainty to make better inferences.

Keywords: regression analysis, uncertainty, qualitative data, statistical distributions

\section{Introduction}

Research methods are commonly divided into quantitative and qualitative methods. Quantitative methods rely on the law of large numbers and express tendencies. Uncertainty is generally expressed as 'errors', and it is assumed that these errors on average cancel each other out so that we have measurement and noise, estimates and uncertainty. Qualitative methods, by contrast, tend to be interpretative and rely on ad-hoc analysis to find patterns in usually complex and noisy data. Uncertainty is generally expressed verbally, and it is assumed that researchers are able to distinguish measurement from noise. When samples are small, some researchers rely on qualitative comparative analysis (QCA) in an attempt to bridge the benefits of qualitative and quantitative methods, despite much evidence that this fails to produce reliable results (Lucas and Szatrowski 2014; Krogslund, Choi, and Poertner 2015).

In the social sciences, many of our theories, notions, and hypotheses are vague and difficult to measure (McElreath 2016). If we quantify concepts like 'early career' or 'a newcomer to a society', we hope that measurement errors are not systematic and on average do not bias our findings. In qualitative analysis, we 
hope that we recognize these concepts when we encounter them despite their vague definitions. Moreover, in many situations, we lack the ability to collect large samples - be this for practical, ethical, or other reasons -, or there may be just a few cases to analyse (think of revolutions or economic depressions). This adds to the uncertainty about our inferences. Here I argue that we should formalize our uncertainty about our data, and use regression analysis to draw better inferences, especially when it comes to small $\mathrm{N}$ and qualitative data. ${ }^{1}$ Qualitative data are data that tend to have high levels of uncertainty, but this need not disqualify them from systematic analysis.

The basic intuition is that we replace fuzzy and uncertain data points with credibility distributions. Rather than trying to 'do away' with the errors, we can formalize our uncertainty, which should lead to richer inferences, and allows including data in formal analyses where it is conceptually difficult to agree on a specific datum. With credibility distributions as the starting point, we can then sample from these distributions and create multiple data sets that, when combined, allow inferences that take into account the uncertainty of our measurements - just like we combine multiply imputed data sets in the case of multiple imputations to address missing data (Allison 2001). Contrary to QCA and many typologies, the complexity of the data need not be reduced in arbitrary ways, but can be modelled and incorporated in standard regression analyses (compare this to efforts to analyse textual data quantitatively; Grimmer and Stewart 2013; Benoit et al. 2016). With this, we can clearly isolate qualitative judgements to data generation, and not have qualitative judgements interfere with the analysis of data.

\section{Intuition}

The intuition of this paper is that we can make better (richer) inferences by modelling uncertainty using credibility distributions. Credibility distributions can be used to express uncertainty about measurement (just like subjective priors can be used in Bayesian inference). In the social sciences, often we cannot 'put a number' to concepts we are interested in, and often we should not, because doing so implies less uncertainty than there actually is. While many researchers have given up on systematic quantitative methods because they are unable or unwilling to quantify their data, this need not be the case: Even with the fuzziest and most uncertain data we can normally indicate tendencies. For instance, following an in-depth interview, we can typically indicate whether a person is more or less likely to say participate in a protest, or identify with a particular group. One person may routinely participate in protest, which implies a small

\footnotetext{
${ }^{1}$ Contrary to common usage, I do not consider the number of interviewees to be the appropriate $\mathrm{N}$, but the number of statements about our quantity of interest (which is what is typically coded in computer-aided qualitative data analysis (CAQDA)), with these statements clustered in respondents. This may still leave studies with a small $\mathrm{N}$ and great reliance on prior knowledge.
} 
amount of uncertainty. For another person, it 'really depends', which implies a large amount of uncertainty or a bi-modal distribution. Using a standard statistical distribution, we can formalize the fuzziness and uncertainty of our data (Figure 1).

Beta $(\alpha=1.5, \beta=5)$

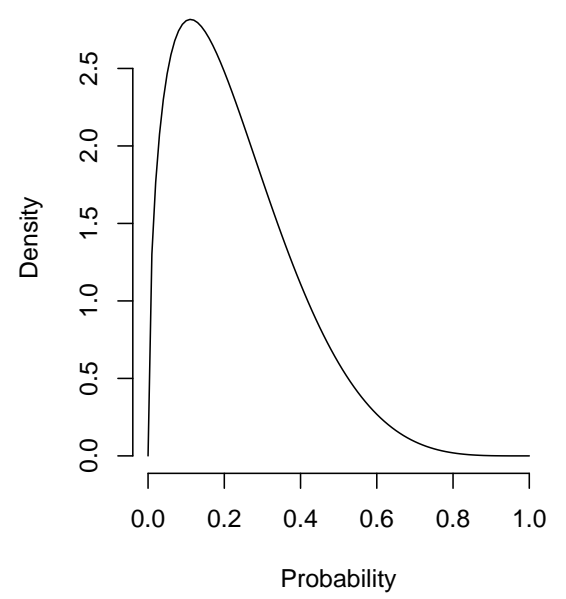

Beta $(\alpha=15, \beta=50)$



Figure 1: Two beta distributions to illustrate credibility distributions: In both panels, there is a tendency towards zero (e.g. 'not interested'); in the panel on the right there is less uncertainty about the position - most values are between around 0.1 and $0.4-$, while in the panel on the left there is more uncertainty indicated by a 'broader' distribution.

Credibility distributions can be used to formalize qualitative statements like we find them in unstructured and semi-structured interview situations, or in unstructured text like newspapers or blog posts. While we cannot put a number on it, we can adequately formalize 'somewhat angry' and 'quite angry'. The credibility distribution is a formal statement of how we interpret the data, without the need to reduce the uncertainty inherent in qualitative data.

By using credibility distributions, we express our uncertainty about the data, and not only at the later stage during analysis. This way we do not rely on assumptions that the 'errors' in the data on average cancel each other out. Such an assumption is particularly problematic for studies with few cases, studies with large uncertainty, and studies where we know that the 'errors' do not cancel each other out (but the assumption that they do is just convenient). Rather than abandoning systematic regression analysis, we can express our uncertainty using credibility distributions and avoid ad-hoc interpretations hoping that - in our capacity as researchers - we can distinguish signal from noise. 


\section{Method}

In a first step, we use statistical distributions to include uncertainty in our data. Rather than quantifying by using numbers (e.g. variable interest $=0$ 'not interested'), we use distributions that specify the uncertainty inherent in the data (Figure 1; e.g. variable interest $=\operatorname{rbeta}(1,1.5,5)){ }^{2}$ There are many statistical distributions that can approximately summarize our uncertainty, including normal, beta, Cauchy, or gamma distributions with appropriate shape parameters. We can use kernel distributions if appropriate, and were we have 'perfectly' measured values, say whether a person pays taxes in a particular country, we can express this certainty using a uniform distribution. I refer to these distributions as credibility distributions.

In a second step, we create multiple data sets by randomly drawing from these credibility distributions. It turns out that the use of credibility distributions to formalize uncertainty is an application multiple imputations, just like it is increasingly used to deal with missing data (Allison 2001). Once we have multiple data sets, we can run regression analysis on each of them, and then combine the results (King et al. 2001).

\section{Possible Applications}

\section{Qualitative indicators}

The outlined procedure may be applied to cases where we have a (vague) definition of the concept, many potential indicators to measure a concept, or difficulty to measure our concepts. For instance, let us assume we are classifying countries, and wish to specify whether countries are democratic. Rather than coding the countries using an arbitrary system, or averaging a systematic set of indicators (typically assuming that each indicator is equally important), we can use credibility distributions to express our uncertainty. Some researchers will probably prefer a set of indicators, others will probably prefer a more 'intuitive' approach - both should be able to express their data in terms of credibility distributions. For those relying on multiple indicators, it is possible to express the uncertainty of each indicator, say 'press freedom', and then combine these distributions (or values) for the overall indication how democratic a country is.

Once we have the countries classified (each with its credibility distribution to summarize our beliefs), we can create multiple data sets that sample from these credibility distributions. We then run regression analysis on each of these data sets, and combine the results just like we do with multiple imputations created

\footnotetext{
${ }^{2}$ This $\mathrm{R}$ code randomly draws one value from a beta distribution with $\alpha=1.5$ and $\beta=5$, the distribution illustrated on the left of Figure 1
} 
Beta $(\alpha=1.5, \beta=5)$

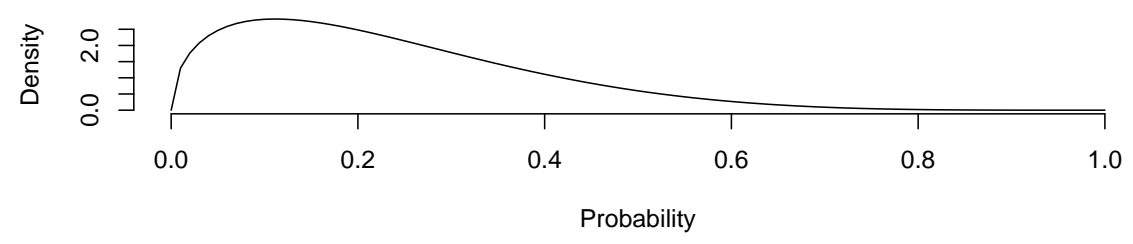

Beta $(\alpha=10.5, \beta=5)$



Figure 2: Two beta distributions to illustrate credibility distributions of how democratic two countries are. In the : In the top panel, there is a tendency towards zero (e.g. 'not democratic'); in the bottom panel there is a tendency towards 1 (e.g. 'democratic'), and less uncertainty about the position. 
to deal with missing data. Particularly for small N studies, a Bayesian framework may bring benefits (Stegmueller 2013), and depending on the researcher, subjective priors may be specified on the assumed association between variables. Whatever the choice, the key point of replacing data 'points' blind to uncertainty with credibility distributions - which formalize our uncertainty - remains, and should lead to better inferences.

\section{Interview data}

Typical interview data are much less structured than qualitative indicators of say the quality of democracy. We are still able to use these data to test associations if we formalize our uncertainty. Say we are interested in the tendency to participate in violent protest, and stipulate that 'anger' has something to do with it. As in other cases, relevant interview data can become overwhelming to analyse, which is why we often rely on computer-aided qualitative data analysis (CAQDA) software to manually code and organize the data. Rather than relying on intuition to identify a relationship and verbal statements to express uncertainty, we can use credibility distributions to classify different instances: Sometimes we might have a clear case of a violent protest, at other times we might not be so sure; sometimes the respondent clearly indicted their anger, at other times there is no mention of emotions - suggesting great uncertainty and a very wide credibility distribution.

\section{Scales}

Uncertainty also plays a role when using scales with data that are more readily quantified. Scales can be useful to combine related variables or capture multiple dimensions of a concept. For instance, we might have a set of variables measuring different aspects of attitudes to redistribution. In such a situation, we typically take the different variables, check if they correlate (drawing on theory, using factor analysis, perhaps throw in a Cronbach alpha), and then simply add the values up. The sum - a single number - is then taken as the 'true' value (as in the most likely estimate), and we run a regression analysis with this scale variable.

By so doing, however, we artificially reduce our uncertainty (probably hoping that the 'errors' would cancel each other out). For instance, let us assume 6 questions on attitudes to redistribution. One person answers $\{3,3,3,3,3,3\}$, the other person answers $\{1,5,1,5,1,5\}$. When averaged, both responses are 3 , but the certainty is quite different. If we were to predict the answer to one of the questions for the first person, we would be quite confident that it is 3 ; in the case of the second person, 3 would also be our 'best' guess, but we would consider different answers quite likely, too. By summarizing responses into credibility 
distributions rather than single measures, we can retain more of our data, which should lead to better inferences.

Different to the previous examples, when it comes to scales, the information in the data may be precise. This means we can use multiple imputations accurately: We can sample from kernel densities.

\section{Software Implementation}

The approach outlined in this paper can be implemented into software. The following two functions are needed: First, a function to replace string variables or numbers with information on distributions (e.g. replace the string "strongly in favour" with "rbeta (a, b)" and appropriate values like $a=10.5$ and $b=5$ as in Figure 2; or replace a single number like 3 with an equivalent credibility distribution). ${ }^{3}$ This way qualitative information can be imported and formalized into credibility distributions. This step of specifying uncertainty is typically a qualitative exercise - and this is exactly what it should be. By using credibility distributions, however, the choices we make are transparent. Put differently, we may (want to, have to) justify our choices. Second, a function to create multiple datasets by randomly drawing from the specified credibility distributions. As multiple imputation, the datasets created will differ, but when results are combined - on 'average' - the specified distributions are respected. ${ }^{4}$

\section{Discussion \& Conclusion}

Currently there is a division between quantitative and qualitative research. Here I argued that this division need not exist, and in many cases qualitative work can benefit from regression analysis and Bayesian analysis. To do so, we need to systematically express our uncertainty about the data - typically large in the unstructured data of qualitative research - which is actually a good thing. Similarly, quantitative research using scales tends to 'hide' the uncertainty inherent in these approaches, and making uncertainty more explicit should lead to richer inferences. ${ }^{5}$

In many cases, what I am propagating is using subjective credibility distributions. While the subjective part of this may seem problematic, I argue that the benefits are clearly larger. By relying on credibility distributions, we end up using more data than those who arbitrarily reduce complexity to fit the data into

\footnotetext{
${ }^{3}$ It might be useful to provide researchers with an interface to help them find the most suitable distribution.

${ }^{4}$ In R, mi, mice, or Amelia objects might be most useful for researchers; ideally we let them choose.

${ }^{5}$ As a side-effect, qualitative researchers drawn to systematic case studies may eventually learn to avoid QCA.
} 
standard regression frameworks (e.g. by using dummy variables with arbitrary cut-offs, coding and combining variables that ignore the uncertainty, or worse relying on unrelaible QCA; Lucas and Szatrowski 2014; Krogslund, Choi, and Poertner 2015). It is important to note that subjective does not mean arbitrary, and with conventional (qualitative) approaches we do not have this assurance. Credibility distributions are formal statements of our uncertainty, and as such can be justified and criticized - which should encourage scientific progress.

By using credibility distributions to express uncertainty, we can isolate our qualitative judgements to the creation of data, and (of course) the interpretation of our data, while the analysis itself need not be 'qualitative. ${ }^{6}$ I have argued that there are also benefits for scales, where we can formalize uncertainty. In all cases - qualitative and quantitative alike -, there should be benefits from using 'all' the data available, which includes information about our uncertainty.

\section{References}

Allison, Paul D. 2001. Missing Data. Quantitative Applications in the Social Sciences (QASS) 136. London: Sage.

Benoit, Kenneth, Drew Conway, Benjamin E. Lauderdale, Michael Laver, and Slava Mikhaylov. 2016. Crowd-sourced Text Analysis: Reproducible and Agile Production of Political Data. American Political Science Review 110, no. 2 (May): 278-95. doi:10.1017/S0003055416000058.

Grimmer, Justin, and Brandon M. Stewart. 2013. Text as Data: The Promise and Pitfalls of Automatic Content Analysis Methods for Political Texts. Political Analysis 21, no. 3: 267-97. doi:10.1093/pan/mps028.

King, G., J. Honaker, A. Joseph, and K. Scheve. 2001. Analyzing incomplete political science data: An alternative algorithm for multiple imputation. American Political Science Review 95, no. 1: 49-69.

Krogslund, Chris, Donghyun Danny Choi, and Mathias Poertner. 2015. Fuzzy Sets on Shaky Ground: Parameter Sensitivity and Confirmation Bias in fsQCA. Political Analysis 23, no. 1 (January): 21-41. doi:10.1093/pan/mpu016.

Lucas, Samuel R., and Alisa Szatrowski. 2014. Qualitative Comparative Analysis in Critical Perspective. Sociological Methodology 44, no. 1 (August): 1-79. doi:10.1177/0081175014532763.

McElreath, Richard. 2016. Statistical Rethinking: A Bayesian Course with Examples in $R$ and Stan. CRC Texts in Statistical Science. Chapman \& Hall.

Stegmueller, Daniel. 2013. How many countries do you need for multilevel modeling? American Journal of Political Science 57, no. 3: 748-61.

\footnotetext{
${ }^{6}$ Future work may identify a Bayesian framework for qualtiative regressions without creating multiple datasets by sampling on the specified credibility distributions directly.
} 
doi:10.1111/ajps.12001. 